\title{
Editorische Hinweise
}

Der Band enthält alle überlieferten Schriften und Artikel sowie literarische Versuche von Engels aus der Zeit bis August 1844 in chronologischer Reihenfolge. Ein Teil der literarischen Versuche wurde bereits im Band 1 der Dritten Abteilung und im Band 1 der Vierten Abteilung der MEGA ${ }^{(2)}$ aufgenommen.

Verwiesen sei besonders auf die in den letzten Jahren aufgefundenen Handschriften und Drucke von Engels, die bisher in MЭC, in „Collected Works" oder in Einzeleditionen veröffentlicht worden sind und im vorliegenden Band erstmals zusammen mit den schon seit Jahrzehnten bekannten Arbeiten geschlossen dargeboten werden. Dazu gehören die Handschrift "Cola di Rienzi", Engels' Beiträge für die Augsburger "Allgemeine Zeitung", die für das "Morgenblatt für gebildete Leser" geschriebene "Erklärung" sowie die Korrespondenzen für den "Northern Star". Der vorliegende Band enthält als Erstveröffentlichung die Gedichtfolge aus dem Jahre 1839 „Beiträge: Impromtu gegen die Geizigen...” sowie die vermutlich im Jahre 1836 entstandenen „Zeichnungen in einem Notizbuch".

Der Anhang ist untergliedert in "Dubiosa" und "Übersetzungen". Während der Arbeit am Band durchgeführte Untersuchungen führten zu dem Ergebnis, daß bei einer Reihe von Artikeln, die in bisherigen Editionen Engels zugeschrieben worden sind, dessen Autorschaft nicht mit ausreichender Sicherheit belegt werden kann. Insgesamt sechs Beiträge, veröffentlicht im "Telegraphen für Deutschland", in der "Rheinischen Zeitung" und im "Northern Star", werden - gleichfalls in chronologischer Reihenfolge - als Dubiosa ediert, einige Artikel werden nicht aufgenommen und einige Artikelteile nur im wissenschaftlichen Apparat mitgeteilt. Die Gründe für diese Entscheidungen werden im Apparatteil „Entstehung 
und Überlieferung” zu den einzelnen Beiträgen bzw. in den „Hinweisen zur Edition" des Apparatteils "Zur publizistischen Arbeit" dargelegt. In dem Teil „Übersetzungen“ wird erstmals eine Übersetzung aus dem Französischen, „Die brasilianische Braut", veröffentlicht, die möglicherweise von Engels stammt.

Maßgeblich für die chronologische Einordnung ist der Beginn bzw. vermutliche Beginn der Niederschrift. In einigen Fällen wird die gesamte Entstehungszeit berücksichtigt, in Einzelfällen werden auch inhaltliche Kriterien für die Anordnung herangezogen. Bei einem großen Teil der im Band veröffentlichten Beiträge konnte die Entstehungszeit erstmals ermittelt bzw. präzisiert werden. In einigen Fällen sind beträchtliche Abweichungen zu früheren Angaben über die Datierung zu verzeichnen. In Fortsetzungen erschienene Artikel werden als ein Werk dargeboten, die einzelnen Beiträge einer Artikelserie jedoch als selbständige Artikel behandelt. Über die Gründe für das jeweils gewählte Verfahren gibt, wenn erforderlich, der wissenschaftliche Apparat Auskunft.

Alle überlieferten autorisierten Textzeugen werden entweder als Grundlage für den Edierten Text oder - auf die abweichenden Stellen verkürzt im Variantenverzeichnis wiedergegeben. In Ausnahmefällen werden auch nicht autorisierte Drucke berücksichtigt. Die Begründung dafür gibt der wissenschaftliche Apparat. In den editorischen Hinweisen zu den einzelnen Arbeiten wird mitgeteilt und, wenn notwendig, erläutert, welche Textzeugen dem Edierten Text zugrunde gelegt und welche im Variantenverzeichnis dargeboten werden.

Der Edierte Text folgt der angegebenen Textgrundlage. Eine Vereinheitlichung oder Modernisierung der Orthographie und Interpunktion wird nicht vorgenommen. Jedoch erfolgt eine Textrevision, um eindeutig fehlerhafte Textstellen zu beseitigen. Eindeutige Schreib- und Druckfehler werden im Edierten Text korrigiert und nicht in das Korrekturenverzeichnis aufgenommen. Sinnverändernde redaktionelle Korrekturen werden stets im Korrekturenverzeichnis ausgewiesen. Fehlerhafte Stellen, deren Korrektur in verschiedenartiger Weise möglich ist bzw. die nicht eindeutig als solche zu bestimmen sind, werden dann im Edierten Text korrigiert, wenn vieles für eine bestimmte Lesung spricht, in unklaren Fällen jedoch nicht bereinigt. Im Korrekturenverzeichnis wird auf beide Verfahren aufmerksam gemacht, beim zweiten Verfahren wird auf mögliche Korrekturen verwiesen. Versehen bei Faktenangaben sowie bei der Schreibweise von Namen, soweit sie eindeutig als solche bestimmbar sind, werden im Edierten Text korrigiert. Diese Berichtigungen werden im Korrekturenverzeichnis ausgewiesen. Ist der Sachverhalt nicht eindeutig, wird keine Veränderung 
vorgenommen, im Korrekturenverzeichnis jedoch darauf hingewiesen. Redaktionelle Hinzufügungen werden in der Herausgeberschrift (Grotesk) gedruckt und in eckigen Klammern eingeschlossen.

Offensichtliche Interpunktionsfehler werden im Edierten Text ohne Kennzeichnung korrigiert, soweit dadurch keine Sinnänderung eintritt. An- und Abführungszeichen sowie halbe An- und Abführungszeichen werden in einheitlicher Weise gesetzt, auch wenn dies von der jeweiligen Textgrundlage abweicht.

Abkürzungen werden ohne Kennzeichnung ausgeschrieben, ausgenommen solche, deren Ausschreibung ungebräuchlich ist. Ein allgemeiner Verweis erfolgt in den editorischen Hinweisen zu den einzelnen Arbeiten. Abkürzungen von Personennamen und übliche Abkürzungen in bibliographischen Angaben (Zitat- und Literaturnachweise von Engels) bleiben bestehen.

Die verschiedenen Hervorhebungsstufen in den handschriftlichen und gedruckten Textgrundlagen werden im Edierten Text einheitlich folgendermaßen wiedergegeben: erste Hervorhebungsstufe - kursiv; zweite Hervorhebungsstufe - gesperrt; dritte Hervorhebungsstufe - kursiv gesperrt. Das Schrift- bzw. Druckbild des zugrunde gelegten Zeugen (Schriftart, Schriftgröße usw.) bleibt unberücksichtigt. Angaben darüber sind in den Zeugenbeschreibungen enthalten.

Bei Handschriften werden Beginn und Ende einer Seite oder eines Bogens kenntlich gemacht, und die Paginierung wird - wenn vorhanden mitgeteilt (siehe Verzeichnis der Abkürzungen, Siglen und Zeichen). Wurde die Paginierung der Handschriften redaktionell ergänzt, so wird die Seitenzahl in eckige Klammern gesetzt. Bei Drucken wird der Seitenwechsel angegeben. Liegt ein Zeitungsdruck zugrunde, erfolgt keine Angabe des Seiten- bzw. Spaltenwechsels; zu Beginn des betreffenden Edierten Textes werden Nummer und Datum der Zeitung vermerkt.

Über einige Besonderheiten der Textgrundlagen sowie deren Wiedergabe im Edierten Text (z. B. die Artikel aus den „Deutsch-Französischen Jahrbüchern" und aus dem „Vorwärts!") geben die „Hinweise zur Edition“ des Apparatteils ,Zur publizistischen Arbeit" Auskunft.

$\mathrm{Zu}$ jeder in den Band aufgenommenen Arbeit wird ein wissenschaftlicher Apparat geboten. Er besteht aus dem Teil Entstehung und Überlieferung (einschließlich Zeugenbeschreibung und Begründung des editorischen Verfahrens), gegebenenfalls aus dem Variantenverzeichnis, dem Korrekturenverzeichnis und den Erläuterungen (siehe dazu auch die Abschnitte VIII und IX des Vorworts zur Gesamtausgabe im Band 1 der Ersten Abteilung). In der Kopfleiste werden die Entstehungszeit sowie die Seitenzahlen des betreffenden Edierten Textes mitgeteilt. 
$\mathrm{Zu}$ einigen Gruppen von publizistischen Arbeiten werden Angaben über die Entstehung, den Charakter, die Erscheinungsweise, die zeitgenössische Wirkung und die Überlieferung der Zeitung oder Zeitschrift unter dem Titel „Zur publizistischen Arbeit” gegeben, in der Regel für den Zeitraum der Mitarbeit von Engels. Aufgenommen werden weiterhin Informationen über das Zustandekommen, den Umfang und die Bedeutung von Engels' Mitarbeit, über mögliche Eingriffe der Redaktion usw. Hier werden auch solche Angaben zur Zeugenbeschreibung und zum editorischen Herangehen vermittelt, die mehr oder weniger auf alle zur betreffenden Gruppe gehörenden Arbeiten zutreffen. Berücksichtigt werden die Zeitungen und Zeitschriften, deren ständiger Mitarbeiter oder Korrespondent Engels war oder in deren Redaktion er mitwirkte. Das betrifft folgende: Telegraph für Deutschland, Morgenblatt für gebildete Leser, Allgemeine Zeitung, Rheinische Zeitung, Schweizerischer Republikaner, The New Moral World, Deutsch-Französische Jahrbücher, Vorwärts! und The Northern Star. Die Charakterisierung aller anderen Zeitungen und Zeitschriften, an denen Engels ebenfalls mitarbeitete, erfolgt in Sammeltextgeschichten zu Beginn des jeweils ersten Artikels.

Der wissenschaftliche Apparat zu jeder einzelnen Arbeit beginnt mit der Darlegung ihrer Entstehung und Überlieferung; von der jeweiligen Wirkungsgeschichte wird nur das unmittelbare zeitgenössische Echo erfaßt. Informationen, die auf eine Gruppe von Arbeiten oder auf die gesamte Artikelserie zutreffen, werden zu Beginn des jeweils ersten Beitrags in Form von Sammeltextgeschichten dargeboten. Bei Handschriften, die zu Lebzeiten von Engels nicht veröffentlicht wurden, wird auf die Erstveröffentlichung und bei anonym erschienenen Artikeln, für welche Engels' Autorschaft nicht durch direkte Beweise belegt werden kann, wird auf die erstmalige Veröffentlichung unter Engels' Namen verwiesen.

In den Zeugenbeschreibungen werden alle dort aufgenommenen Zeugen mit einer Sigle (siehe Verzeichnis der Abkürzungen, Siglen und Zeichen) und zusätzlich mit Zahlenexponenten versehen. Diese Numerierung erfolgt unabhängig vom Charakter der einzelnen Zeugen fortlaufend in der Reihenfolge ihrer Entstehung (2.B. J', $\mathbf{k}^{2}, D^{3}$ ). Nicht autorisierte Zeugen werden mit kleinen Buchstaben bezeichnet. Nicht überlieferte Textzeugen werden nur in besonderen Fällen aufgenommen. Sie werden dann mit der Sigle $X$ bzw. $X$ verzeichnet und erhalten eine gesonderte Zählung.

Das Variantenverzeichnis enthält alle von Engels vorgenommenen Textänderungen, die den Text inhaltlich oder stilistisch weiterentwickeln. Diese Varianten treten auf als Textreduzierungen (Tilgungen nicht korrupter Textstellen), Textergänzungen (Einfügungen, Zusätze), Textersetzungen und Textumstellungen. Nicht verzeichnet werden folgende Textänderun- 
gen: von Engels korrigierte Schreib- oder Druckfehler; von Engels vorgenommene Veränderungen der Orthographie oder der Interpunktion, die keinen Einfluß auf die Sinngebung haben; Schreibansätze, die keinen erkennbaren Sinn ergeben oder bei denen der Sinn der von Engels beabsichtigten Aussage nicht wenigstens mit Wahrscheinlichkeit rekonstruiert werden kann; innerhandschriftliche Sofortkorrekturen, die formale Berichtigungen grammatischer oder stilistischer Versehen darstellen, jedoch weder die inhaltliche Aussage des Textes verändern noch den Stil der gesamten Darstellung modifizieren.

Das Variantenverzeichnis ist ein mit notwendigen Stützworten (aus dem Edierten Text) versehener Werkstellenapparat, d. h., es verzeichnet von Werkstelle zu Werkstelle fortschreitend alle Varianten einer Textstelle, die innerhalb eines oder in mehreren Textzeugen überliefert sind. Die innerhandschriftlichen Varianten zu einer Werkstelle werden entweder mit Hilfe diakritischer Zeichen hintereinander oder mit der Methode der Zeilenparallelisierung untereinander dargeboten. Das Variantenverzeichnis benutzt eine im wesentlichen diskursive Verzeichnungsform, d.h., es wird der Inhalt der Textveränderungen festgehalten, jedoch nicht die Form, in der diese Änderungen durchgeführt wurden.

Die Varianten werden mit Hilfe diakritischer Zeichen dargestellt (siehe Verzeichnis der Abkürzungen, Siglen und Zeichen). Sofortvarianten treten häufig in Form von „Abbrechungen“ auf, d.h. als Textänderungen, bei denen Engels die Gedankenführung unterbrach und ihr (meist durch Tilgung, aber auch durch Ersetzung von Wörtern oder Wortteilen, Änderungen von Flexionsendungen und Einfügungen) einen neuen Verlauf gab. Abbrechungen, die in der Handschrift vollständig getilgt wurden, werden folgendermaßen dargestellt: Nach dem Stützwort aus dem Edierten Text folgt in Winkelklammern der getilgte Passus und danach das Abbrechungszeichen. Abbrechungen, bei denen Teile des Wortbestandes in die nächste Schicht übernommen wurden, werden im Prinzip genauso dargestellt. $\mathrm{Da}$ in diesen Fällen oft nicht sicher zu erkennen ist, an welcher Stelle des Satzes der Autor abbrach und änderte, wird das Abbrechungszeichen in der Regel an die Stelle gesetzt, an der spätestens die Textumformung erfolgt sein muß; der in Winkelklammern stehende Text des ursprünglichen, abgebrochenen Satzverlaufs umfaßt in diesen Fällen also auch Wörter oder Wortteile, die in der Handschrift nicht getilgt, sondern in die neue Textfassung einbezogen wurden. Die Winkelklammern kennzeichnen hier ein im Ganzen verworfenes Textstück.

Bei den überlieferten Druckvorlagen in Engels' Handschrift, die Eingriffe eines Redakteurs enthalten, werden auch letztere zusammen mit den Varianten in einem Verzeichnis dargeboten, das dann den Titel „Verzeich- 
nis der Varianten und redaktionellen Eingriffe" trägt. Bei dem Beitrag "Die Beduinen" (S. 22/23) wird das "Verzeichnis der redaktionellen Eingriffe" an Hand einer von Engels angefertigten Abschrift rekonstruiert.

Die Erläuterungen geben alle für das Verständnis des Textes (einschließlich der Varianten) erforderlichen Erklärungen und Hinweise, soweit dies nicht schon im Apparatteil „Entstehung und Überlieferung" geschehen ist. Sie bieten ferner die Übersetzung lateinischer und griechischer Textstellen. Wichtiger Bestandteil der Erläuterungen ist der Nachweis der von Engels benutzten Literatur. Wenn nicht ermittelt werden konnte, welche Ausgabe Engels benutzt hatte, erfolgt in den Erläuterungen ein entsprechender Verweis. Konnte von der von Engels benutzten Quelle kein Exemplar beschafft werden, werden die Quellen nach Nachschlagewerken bibliographiert, und es wird darauf verwiesen. Bei Zitaten aus der Weltliteratur wird in der Regel auf die Angabe einer konkreten Ausgabe verzichtet. Abweichungen zwischen Engels' Zitierweise und der benutzten Quelle werden verzeichnet, wenn diese inhaltlich belangvoll oder für eine vorgenommene oder mögliche Textrevision von Bedeutung sind. Außerdem werden alle von Engels gegenüber der zitierten Quelle vorgenommenen Hervorhebungen mitgeteilt.

Verweisungen auf bereits vorliegende Bände der MEGA ${ }^{(2)}$ erfolgen unter Verwendung der im Verzeichnis der Abkürzungen entschlüsselten Siglen. Bei Zitaten aus Arbeiten von Marx und Engels, die in MEGA(2) noch nicht erschienen sind, wird direkt auf den Erstdruck verwiesen.

Der wissenschaftliche Apparat enthält außerdem ein „Verzeichnis der nicht überlieferten Arbeiten" und ein „Verzeichnis der bereits in anderen MEGA ${ }^{2}$-Bänden enthaltenen literarischen Arbeiten" von Engels aus der Zeit bis August 1844.

Die Register erfassen den Edierten Text und die Varianten. Das Literaturregister umfaßt alle Literatur (Bücher, Broschüren, Zeitschriftenaufsätze, Zeitungsartikel, Dokumente usw.), die in den Texten direkt oder indirekt zitiert bzw. direkt oder indirekt erwähnt wird. Die Titel werden alphabetisch nach Verfassernamen, die Titel anonymer Veröffentlichungen nach dem ersten Wort, das kein bestimmter oder unbestimmter Artikel ist, eingeordnet. Ist kein Titel vorhanden, werden die ersten Worte des Textes mit Auslassungspunkten angeführt. Wird der Titel aus der Inhaltsübersicht übernommen, wird dieser in runde Klammern gesetzt. Nicht aufgenommen werden allgemeine Hinweise auf Verträge, Verfassungen, Gesetze u.ä. sowie auf Manuskripte, Archivmaterialien und Briefe, die zum Zeitpunkt der Abfassung des Textes noch unveröffentlicht waren und es zum Teil auch heute noch sind. 
Das Namenregister stellt die in den Texten direkt oder indirekt genannten Personennamen zusammen, wobei literarische und mythologische Namen einbezogen werden. Aufgenommen werden auch die Verfasser von Veröffentlichungen, die im Text selbst nicht genannt, deren Arbeiten aber direkt oder indirekt erwähnt oder zitiert werden. Die alphabetische Einordnung der Namen erfolgt nach ihrer authentischen Schreibweise, bei griechischen und kyrillischen Buchstaben nach der entsprechenden transkribierten Form. Alle von der authentischen Form abweichenden Schreibweisen des Edierten Textes werden im Register der authentischen Schreibweise in runden Klammern zugefügt und, wenn notwendig, gesondert als Verweisung angeführt. Verschlüsselte Namen im Edierten Text sind in Erläuterungen erklärt.

Das Sachregister umfaßt die Begriffe, die den wesentlichen Inhalt der Arbeiten von Engels und die Entwicklung seiner Auffassungen bis August 1844 widerspiegeln. Es ist im Prinzip in der Redaktionssprache und in moderner Orthographie abgefaßt. Die Schlagworte sind unmittelbar dem Edierten Text entnommen oder lehnen sich weitgehend an diesen an. Daher werden sie in einigen Fällen in der Sprache des jeweiligen Originals gegeben bzw. steht der originalsprachige Begriff in Klammern hinter dem Schlagwort in der Redaktionssprache.

Der vorliegende Band wurde bearbeitet von lleana Bauer (Leitung und Bearbeitung der bis Oktober 1842 entstandenen Beiträge), Inge Taubert (Bearbeitung der zwischen November 1842 und Ende August 1844 entstandenen Beiträge), Anita Springer-Liepert (Bearbeitung eines Teiles der bis Oktober 1842 entstandenen Beiträge) und Ingrid Donner (Fertigstellung der Texte, Varianten- und Korrekturenverzeichnisse). An der Bearbeitung oder Vorbereitung der Beiträge des gesamten Bandes wirkten mit Ingrid Donner, Johanna Dehnert und Elke Röllig. Das Namenregister wurde zusammengestellt von Ingrid Donner, das Sachregister von Jutta Nesler und das Literaturregister von Christa Krause. Die wissenschaftlichtechnischen Arbeiten wurden von Christa Krause und Jelena Butter durchgeführt.

Der Band wurde seitens der Redaktionskommission betreut und begutachtet von Inge Taubert. Gutachter des IML beim ZK der KPdSU waren Nikita Fedorowski und Irina Schikanjan. Teilgutachten zu einzelnen Arbeiten sowie Stellungnahmen zu bestimmten Sachgebieten erfolgten durch Helmut Bock (Berlin), Hans-Otto Dill (Berlin), Werner Feudel (Berlin), Manfred Hessel (Warnemünde), Leonard Jones (Halle), Ursula Kändler (Leipzig), Michael Knieriem (Wuppertal), Rudolf Koschulla (Leipzig), Hansulrich Labuske (Berlin), Kurt Müller (Berlin), Henri Poschmann (Weimar), 
Elfriede Rehbein (Dresden), Reinhard Schaffer (Warnemünde), Anneliese Schmitt (Berlin) und Waltraud Woeller (Berlin).

Die Herausgeber danken allen wissenschaftlichen Einrichtungen und Privatpersonen, die bei der Vorbereitung des Bandes Unterstützung gewährten. Die Einsichtnahme in die Originale von Engels ermöglichten: das Historische Archiv der Stadt Köln, das Deutsche Literaturarchiv/ Schiller-Nationalmuseum Marbach a. N., der Bergische Geschichtsverein und das Engels-Haus Wuppertal. Archivmaterialien stellten zur Verfügung das Zentrale Staatsarchiv Potsdam, Dienststelle Merseburg, das Staatsarchiv des Kantons Zürich, die Zentralbibliothek Zürich und das Staatsarchiv Hamburg. Die Deutsche Staatsbibliothek und die Universitätsbibliothek Berlin unterstütten die Arbeit durch Ausleihe zahlreicher Bücher und Periodica aus ihren Beständen. 